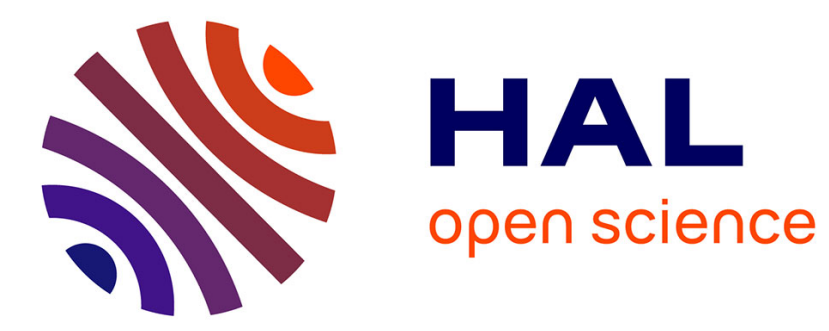

\title{
Water nanodroplets confined in zeolite pores
}

François-Xavier Coudert, Fabien Cailliez, Rodolphe Vuilleumier, Alain Fuchs, Anne Boutin

\section{To cite this version:}

François-Xavier Coudert, Fabien Cailliez, Rodolphe Vuilleumier, Alain Fuchs, Anne Boutin. Water nanodroplets confined in zeolite pores. Faraday Discussions, 2009, 141, pp.377-398. $10.1039 / \mathrm{b} 804992 \mathrm{k}$. hal-02116837

\section{HAL Id: hal-02116837 https://hal.science/hal-02116837}

Submitted on 1 May 2019

HAL is a multi-disciplinary open access archive for the deposit and dissemination of scientific research documents, whether they are published or not. The documents may come from teaching and research institutions in France or abroad, or from public or private research centers.
L'archive ouverte pluridisciplinaire HAL, est destinée au dépôt et à la diffusion de documents scientifiques de niveau recherche, publiés ou non, émanant des établissements d'enseignement et de recherche français ou étrangers, des laboratoires publics ou privés. 


\title{
Water nanodroplets confined in zeolite pores
}

\author{
François-Xavier Coudert ${ }^{a *}$, Fabien Cailliez $^{b}$, \\ Rodolphe Vuilleumier ${ }^{c \dagger}$, Alain H. Fuchs ${ }^{b}$ and Anne Boutin ${ }^{a \ddagger}$
}

\author{
${ }^{a}$ Laboratoire de Chimie Physique, \\ Univ Paris-Sud, F-91405 Orsay, France. \\ ${ }^{b}$ École Nationale Supérieure de Chimie de Paris (Chimie ParisTech) \\ and Université Pierre et Marie Curie Paris 6, F-75005 Paris, France. \\ ${ }^{c}$ Laboratoire de Physique Théorique de la Matière Condensée, \\ Université Pierre et Marie Curie Paris 6, F-75005 Paris, France.
}

May 1, 2019

\begin{abstract}
We provide a comprehensive depiction of the behaviour of a nanodroplet of $\simeq 20$ water molecules confined in the pores of a series of 3D-connected isostructural zeolites with varying acidity, by means of molecular simulations. Both Grand Canonical Monte Carlo simulations using classical interatomic forcefields and first-principles Car-Parrinello molecular dynamics were used in order to characterise the behaviour of confined water by computing a range of properties, from thermodynamic quantities to electronic properties such as dipole moment, including structural and dynamical information. From the thermodynamic point of view, we have identified the all-silica zeolite as hydrophobic, and the cationic zeolites as hydrophilic; the condensation transition in the first case was demonstrated to be of first order. Furthermore, in-depth analysis of the dynamical and electronic properties of water showed that water in the hydrophobic zeolite behaves as a nanodroplet trying to close its hydrogen bond network onto itself, with a few short-lived dangling $\mathrm{OH}$ groups, while water in hydrophilic zeolites "opens up" to form weak hydrogen bonds with the zeolite oxygen atoms. Finally, the dipole moment of confined water is studied and the contributions of water self-polarisation and the zeolite electric field are discussed.
\end{abstract}

\footnotetext{
*Present address: University College London, Gower Street, London WC1E 6BT, United Kingdom.

${ }^{\dagger}$ Present address: Département de Chimie, École Normale Supérieure, 75005 Paris, France.

${ }^{\ddagger}$ E-mail: anne.boutin@lcp.u-psud.fr
} 


\section{Introduction}

Water in zeolites represents a model system for a wide range of experimental as well as theoretical investigations, aimed at understanding the effect of confinement on the structure,[1] dynamics[2, 3] and thermodynamics[4, 5, 6, 7, 8] of molecular fluids. Understanding the behaviour of nano-confined water is also relevant to such important problems as protein stability, $[9,10,11,12,13]$ transport in ion channels, $[14,15,16]$ nano-fluidic devices[17] and the thermodynamics of colloidal assemblies.[18, 19] From a practical point of view, water plays a key role in many applications of zeolites such as ion-exchange and liquid phase separation.

Several computer simulation studies of water in zeolites have been reported in the past few years, using a variety of techniques including energy minimisation (with effective shell model-ionic potentials),[20, 21, 22, 23, 24] Car-Parrinello first-principles Molecular Dynamics (MD), [25, 26, 27, 28, 29] classical MD or Monte Carlo (MC) simulations using conventional two-body or polarisable forcefields. $[7,8,30,31,32,33,34$, $35,36,37,38,39]$

Here we report, for the first time to our knowledge, a comprehensive description of the behaviour of 20 water molecules confined in the pores of a series of 3D-connected isostructural zeolites with varying acidity, by means of both classical Grand Canonical MC simulations and first-principles Car-Parrinello MD simulations. We characterise the behaviour of confined water by a range of properties, from thermodynamic quantities to electronic properties such as dipole moment, including structural and dynamical information.

\section{Methods and systems}

\subsection{Systems studied}

To understand the behaviour of water confined in the nanopores of a zeolite and the influence of the cationic content (or acidity) of the zeolite on the properties of water, we have studied three zeolites with the same structure and different Si:Al ratios. The chosen system was the LTA structure type, which displays supercages (also called $\alpha$ cages) of diameter $\simeq 13 \AA$, connected to one another in a cubic symmetry by 8-ring windows of diameter $\simeq 6 \AA$, and smaller sodalite cages of diameter $\simeq 7 \AA$ (see Figure 1 ). As opposed to previous simulations of $1 \mathrm{D}$ water chains confined in zeolitic materials such as Li-ABW, bikitaite, $[25,26]$ and VPI-5, [27] the medium-size, 3D-connected pores of LTA make it a good candidate for studying the effect of confinement on liquid water condensation in open framework nanoporous materials. Furthermore, the small size of the LTA unit cell (approximately $12 \AA$ ) makes it a reasonable system for first-principles molecular dynamics simulations.

Synthesis of all-silica zeolites remains an experimental challenge. Although a synthesis of the reportedly all-silica LTA zeolite was published in the 1970's,[40] the proposed procedure (thermal dealumination) is known to result in materials exhibiting nonnegligible concentrations of defects[41] and no direct structural characterisation was reported in this study. To our knowledge, this synthesis was never reproduced. More recently, the all-silica LTA zeolite was synthesised and characterised by the groups of Corma[42] and Patarin.[43, 44] Because the work reported in the present communication was started before these experimental results where published, the structure we 
used is an hypothetical structure taken from Ref. [45]. Its unit cell structure $\left(\mathrm{Si}_{24} \mathrm{O}_{48}\right)$ is cubic $(P m \overline{3} m$ symmetry and $a=11.9 \AA)$ and contains one supercage and one sodalite cage. It is very similar in both unit cell parameter and atomic positions to the experimental structures reported by Patarin and Corma.

When it comes to cationic zeolites with the LTA structure, a large number of materials have been synthesised. Most natural and synthetic zeolites feature sodium as an extraframework cation. We have thus studied two different cationic LTA zeolites, containing 1 and 9 sodium cations respectively. The unit cell formulas are $\mathrm{NaSi}_{23} \mathrm{O}_{48} \mathrm{Al}$ and $\mathrm{Na}_{9} \mathrm{Si}_{15} \mathrm{O}_{48} \mathrm{Al}_{9}$, corresponding to $\mathrm{Si}: \mathrm{Al}$ ratios of 23 and 1.67 respectively. To make our discussion easier to read, these two materials will hereafter be named $\mathrm{Na}_{1}$-LTA and $\mathrm{Na}_{9}$-LTA. $\mathrm{Na}_{1}$-LTA corresponds to a single aluminium atom and a single $\mathrm{Na}^{+}$cation per unit cell. As all T atoms* in the LTA structure are symmetrically equivalent, there is only one possible framework structure for this material, which is represented in Figure 2. As there has been no synthesis of a LTA zeolite with this particular Si:Al ratio, we have used the same structure and unit cell parameter as the all-silica LTA zeolite mentioned above.

The choice of Nag-LTA for the present study was dictated by our intention to model a zeolite with a high cationic content (low Si:Al ratio) and the constraints of the first-principles approach. This latter arises from the Loewenstein rule,[46] which states that no two aluminium atoms of a zeolite structure should be linked directly by an oxygen atom. This results in highly cationic zeolites having unit cells multiple times larger than the unit cell of the LTA structure, which are then too costly to perform first-principles molecular dynamics simulations. For example, the unit cell parameter of the well-known NaA zeolite $\left(\mathrm{Na}_{12} \mathrm{Si}_{12} \mathrm{Al}_{12} \mathrm{O}_{48}, \mathrm{Si}: \mathrm{Al}=1\right)$ is twice that of the pure-silica LTA structure. We thus performed a systematic exploration of all possible distributions of $n$ aluminium atoms among the $24 \mathrm{~T}$ atoms of a single LTA unit cell that conform to the Loewenstein rule. These calculations proved that the only values of $n$ compatible with both constraints are in the range $1 \leq n \leq 9$. They furthermore showed that for $n=9$, corresponding to the lowest Si:Al ratio, there are only two possible distributions. These two structures, though not symmetrically equivalent, are very similar (see Figure 1S). We have thus taken the first one as a starting point for our simulations, along with a unit cell parameter taken from the experimental structure of zeolite $\mathrm{NaA}(a=11.26 \AA)$.[47]

\subsection{Monte Carlo simulations}

\subsubsection{Models and forcefields.}

The development of models and forcefields to describe the interaction of zeolites with various guest molecules is a very active field of research.[48] In this study, the focus is put on the properties of confined water and their evolution with the nature of the zeolite. No chemical change occurs during water condensation in any of the LTA zeolites studied and Monte Carlo simulations were thus performed in the classical limit. We used existing forcefields, previously designed in the group of Fuchs and known to yield good transferability for both all-silica[7, 49] and cationic zeolites.[50] For Na-LTA and Nag-LTA systems, an average tetrahedrally bonded (T) atom was used, instead of distinguishing aluminium and silicium atoms.[50] It has been shown that this yields a reasonable description of sodium cation distributions in faujasites.[50, 51]

\footnotetext{
${ }^{*}$ In a zeolite framework, the tetrahedrally bonded $\mathrm{Si}$ and $\mathrm{Al}$ atoms are referred to as the $\mathrm{T}$ atoms.
} 
The zeolite framework was considered fixed. Its interaction with extraframework cations and guest molecules included a Coulombic term and a repulsion-dispersion term; the latter was modeled by a Buckingham (or exp-6) potential for the cations[52] and a Lennard-Jones potential[50] for water molecules. A summary of the forcefield parameters (partial charges, Lennard-Jones and Buckingham parameters) can be found in Tables 1 and 2). The rigid, nonpolarisable TIP4P model[53] was used for water. Lorentz-Berthelot rules have been used to obtain cross potential parameters.

\subsubsection{Grand Canonical Monte Carlo simulations.}

Adsorption isotherms were obtained using Grand Canonical Monte Carlo simulations[54, $55]$ to compute the average number of adsorbed water molecules for several values of the chemical potential. The chemical potential of water was related to the thermodynamic pressure by using the ideal gas law in the vapour phase, and by computing the $\mu(P)$ relation in liquid water as described in Ref. [7]. Preinsertion, [56] orientation[57, 58] and jump[50] bias moves were used to accelerate the convergence of the Monte Carlo simulations.

All Monte Carlo runs were performed at $300 \mathrm{~K}$ on a $2 \times 2 \times 2$ supercell to minimise the influence of periodic boundary conditions. Typical runs included 10 to 200 million steps to equilibrate the system followed by a production run of 30 to 100 million steps. Longrange electrostatic interactions were taken into account using the Ewald summation technique. In order to improve the efficiency of the calculations, the electrostatic and repulsion-dispersion interaction energies between the rigid zeolite framework and both water molecules and extraframework cations were precomputed on a grid (with a grid mesh of $0.2 \AA$ ) and stored for use during the simulation.

\subsubsection{Grand Potential Computation.}

The question of the nature of the transition in adsorption/desorption experiments is often subject to debate. Is this transition a real phase transition, between a gas-like and a liquid-like fluid? Likewise, when hysteresis occurs, its origin also raises several questions. Is it due to a bad sampling of the system or can it be related to the existence of multiple metastable states? In order to bring an answer to these issues, knowledge of the thermodynamic potential of the system is necessary. Such a quantity is not straightforward to obtain and requires the use of sophisticated techniques. Recently, Wang and Landau developed such a technique that is based on the computation of the density of states (DOS) on-the-fly, using biased Monte Carlo simulations.[59, 60] (Once the DOS is known, the calculation of the thermodynamic potential is straightforward.) This method has been used successfully to study a wide range of systems, including Ising models,[59] Lennard-Jones fluids[61] and clusters,[62] or proteins.[62, 63] It has also been revisited in the context of expanded ensembles.[64, 65, 66] In expanded ensembles, substates of the system are defined by the introduction of a new variable often called reaction coordinate $\xi$. The aim of those EXEDOS (Expanded Ensemble Density Of States) simulations is not to determine the density of states as a function of the energy, like in the original Wang-Landau algorithm, but as a function of the reaction coordinate, in order to compute variations of thermodynamic quantities along $\xi$. When dealing with adsorption, the thermodynamic potential is the Grand Potential $\Omega$, since simulations are conducted in the Grand Canonical ensemble. We have applied the EXEDOS methodology to compute $\Omega$ as a function of the number of adsorbed water molecules in the zeolite framework. To this end, we have computed the density of states of the system as a function of $N$, noted $Q(N)$. 
The probability to generate randomly a configuration with $N$ water molecules is proportional to $Q(N)$. A flat distribution is thus obtained if each state is visited with probability proportional to $1 / Q(N)$. The acceptance probabilities of insertion and destruction moves have been modified accordingly (rotations, translations, and jump moves are still accepted according to the conventional Metropolis criteria):

$$
\begin{gathered}
p(N \rightarrow N+1)=\min \left(1, \frac{V}{(N+1) \Lambda^{3}} \frac{Q(N)}{Q(N+1)} e^{-\beta \Delta E}\right) \\
p(N \rightarrow N-1)=\min \left(1, \frac{N \Lambda^{3}}{V} \frac{Q(N)}{Q(N-1)} e^{-\beta \Delta E}\right)
\end{gathered}
$$

$\Lambda$ is the de Broglie wavelength and $\Delta E$ is the energy change between the new and the old configuration. $Q(N)$ is determined iteratively during the simulation. Initially, $Q$ is uniformly set to 1 for the entire range of values accepted for $N$. $Q$ is then updated at each insertion or destruction trial move between two configurations containing respectively $N_{1}$ and $N_{2}$ water molecules. If the move is accepted, $Q\left(N_{2}\right)$ is multiplied by a factor $f$ (with $f>1$ ) ; if the move is rejected, $Q\left(N_{1}\right)$ is multiplied by $f$. At the same time, a $N$-histogram $H(N)$ is accumulated. The simulation is stopped when each bin has been visited at least 100/ $\sqrt{\ln f}$ times, following Zhou and Batt.[67] At this time, the multiplicative factor is decreased, so that $f \rightarrow \sqrt{f}$. The histogram is reset to zero and a new cycle is started, until the histogram is flat again. This process is repeated until $f$ reaches a small enough value. $f$ is set to $e^{1}$ for the initial cycle, and 20 cycles are made, so that the last value of $f$ is approximately equal to $e^{10^{-6}}$. For small values of $f$ (typically $f<e^{10^{-4}}$ ), the "minimum visit per bin" criterion may be too restrictive and the simulation is stopped if the total number of MC moves is superior to $200000 / \sqrt{\ln f}$, as suggested by Poulain et al. [62]

Once $Q(N)$ has been determined, one is able to calculate $\Omega$ for the system, using:

$$
\Omega(\mu, V, T)=-k_{B} T \ln \left(\sum_{N} Q(N) e^{\beta \mu N}\right)
$$

For a given value of the chemical potential $\mu$, the Landau free energy between two states containing respectively $N_{1}$ and $N_{2}$ molecules is:

$$
\Omega_{\mathrm{L}}\left(\mu, N_{2}\right)-\Omega_{\mathrm{L}}\left(\mu, N_{1}\right)=-k_{B} T \ln \left(\frac{Q\left(N_{2}\right)}{Q\left(N_{1}\right)} e^{\beta \mu\left(N_{2}-N_{1}\right)}\right)
$$

This allows to determine $\Omega_{\mathrm{L}}$ to within an additive constant. However, in our particular case, we can determine this constant, since we know that $N=0$ only corresponds to one configuration for any value of $\mu$, so that $\Omega_{\mathrm{L}}(\mu, 0)=0 \mathrm{~kJ} . \mathrm{mol}^{-1}$. Using the $\mu(P)$ relationship for bulk water, we can also present variations of $\Omega$ (or $\Omega_{\mathrm{L}}$ ) as a function of the external pressure of the reservoir.

\section{$2.3 \quad$ First-principles simulations}

\subsubsection{Molecular dynamics.}

The molecular dynamics of anhydrous and hydrated zeolite were performed with the Car-Parrinello approach[68] as implemented in the CPMD software package,[69] using the Kohn-Sham formulation of DFT. We employed the gradient-corrected BLYP 
functional.[70, 71] A plane-wave basis set with an energy cutoff of 70 Ry was used and only the valence electrons were explicitly considered, with semilocal norm-conserving Trouillier-Martins pseudopotentials taking into account the interactions between core and valence electrons. All molecular simulations were performed using periodic boundary conditions on a single cubic unit cell of the zeolite. Hydrogen atoms were deuterated in order to improve decoupling of the dynamics of the ionic and electronic subsystems and allow for a larger time step in the integration of the equations of motion. A timestep of 7 a.u. (0.17 fs) and a fictitious electron mass of 1000 a.u. were used.

Molecular dynamics simulations of the anhydrous zeolite were started from the hypothetical structure for the purely siliceous LTA zeolite available in the literature.[45] For the hydrated zeolites, starting configurations were extracted from the previously performed Grand Canonical Monte Carlo simulations. A first equilibration run of at least $1.2 \mathrm{ps}$ was performed on each system, during which the temperature was controlled to $300 \mathrm{~K}$ by a Nosé-Hoover thermostat[72, 73] with a frequency of $3800 \mathrm{~cm}^{-1}$. Production runs of 5 to $6 \mathrm{ps}$ in the microcanonical NVE ensemble were then performed, from which were extracted all the quantities reported in this article.

\subsubsection{Analysis of electronic properties.}

The total polarisability $M(t)$ of the periodic unit cell was defined using the Berry phase approach of Resta.[74, 75] The IR absorption coefficient of the system, $\alpha(\omega)$, was then computed by calculating the autocorrelation function of the polarisability, within the framework of linear response theory:

$$
\alpha(\omega) \propto \frac{\omega \tanh (\beta \hbar \omega / 2)}{n(\omega) V} \int_{-\infty}^{\infty} d t e^{-i \omega t}\left\langle\sum_{i j} \mu_{i}(t) \mu_{j}(0)\right\rangle
$$

where $n(\omega)$ is the refractive index. A harmonic quantum-classical correction was then applied to the classical correlation function to account for the quantum aspects of the hydrogen motion, as was shown to be adequate in previous works.[76, 77, 78] The resulting infrared spectrum was then smoothed by a linear filter as in Ref. [77, 78].

Dipole moments of individual water molecules were also computed: in order to do so, the electronic wavefunctions are replaced by the maximally localised Wannier functions[79] and the Wannier centers are assigned to each water molecule. [80, 81, 77] The calculation of both the macroscopic polarisability and the Wannier functions was performed every $1.7 \mathrm{fs}$ of the dynamics, for each system.

To characterise the presence of hydrogen bonds and their strength from an electronic point of view, we propose to study atomic polar tensors on hydrogen atoms. These tensors and particularly their strong anisotropy can be shown to be a signature of hydrogen bonding in liquid water.[82] The atomic polar tensor of a given atom $n$ is defined as

$$
\alpha_{i j}^{(n)}=\frac{\partial M_{i}}{\partial R_{n, i}}, \text { where } i \text { and } j \text { can be } x, y \text { or } z
$$

where $M_{i}$ is the component of the total polarisation of the system along axis $i$ and $R_{n}$ is the position of atom $n$.

In this study, the atomic polar tensors were calculated in the Density Functional Linear Response Theory,[83] using Putrino et al.[84] implementation in the CPMD code[69] of the linear response to an external electric field. The atomic polar tensors of

all atoms in a given configuration of the system were computed by three calculations of 
the linear response of the system to an external electric field, according to the following formula:

$$
\alpha_{i j}^{(n)}=\frac{\partial F_{j}^{(n)}}{\partial E_{i}}
$$

where $F^{(n)}$ is the force acting on atom $n$ and $E$ is the external electric field. For each system studied, this analysis was performed on 200 configurations extracted at regular interval from the NVE dynamics.

From the APT tensor we derived an atomic charge, namely the Born charge, as the trace of the APT tensor. To better characterise the APT which is a nonsymmetric $3 \times 3$ tensor we also determined the singular values of the APT from singular value decomposition. The three singular values $\left(\xi_{1}, \xi_{2}\right.$ and $\left.\xi_{3}\right)$ have the dimension of a charge and their differences characterise the anisotropy of the atomic polar tensor.

\section{Results and discussion}

\subsection{Thermodynamics of water in LTA zeolites}

\subsubsection{Water adsorption isotherms.}

Water adsorption isotherms for the three different zeolite models are shown in Figure 3. For Nag-LTA, the isotherm is a typical type I isotherm, according to the IUPAC classification scheme,[85] commonly found in the case of hydrophilic aluminosilicate zeolites. It is characterised by a strong water uptake at low pressure (high value of Henry's constant). In contrast, the isotherm for the all-silica zeolite is typically that of water intrusion into an hydrophobic nanoporous solid:[49, 86] a negligible water uptake is observed up to a pressure of $\simeq 100 \mathrm{MPa}$ (i.e. much higher than the saturated vapour pressure of $3500 \mathrm{~Pa}$ ), at which an abrupt condensation transition takes place. Moreover, in that case, a hysteresis is observed: water extrusion takes place at $\simeq 45 \mathrm{MPa}$.

In the case of $\mathrm{Na}_{1}$-LTA, three different stages can be distinguished during water adsorption. At low pressure, 2 to 5 water molecules per unit cell are involved in small clusters around sodium cations, equally distributed in each of the eight unit cells of the simulation box (see Figure 4a). Around $250 \mathrm{~Pa}$, a first transition occurs that corresponds to the filling of half the unit cells of the simulation box (Figure $4 \mathrm{~b}$ ). This is accompanied by a redistribution of the cations, so that 4 unit cells are filled with 2 cations and water, whereas the other 4 are almost empty. This is the stable situation until 2500-3000 $\mathrm{Pa}$ where a second transition occurs leading to the total filling of all the supercages. This transition is accompanied once again by the displacement of sodium cations so that, in the end, the system is homogeneous with each unit cell filled with water and one cation (Figure 4c). It is interesting to point out the importance of simulating eight unit cells instead of one, because it would otherwise not have been possible to observe such cationic redistribution and inhomogeneous pore filling.

The change in shape of the adsorption isotherms from all-silica to $\mathrm{Na}_{9}$-LTA can be regarded as an evolution from a purely hydrophobic nanoporous solid to a hydrophilic one, the intermediate $\mathrm{Na}_{1}$-LTA case being that of a hydrophobic medium containing strong hydrophilic defects. If we ignore the two-step filling mechanism (which is a consequence of the mobility of sodium cations), the adsorption isotherm in $\mathrm{Na}_{1}$-LTA is of type IV: adsorption at low pressures followed by a plateau, and a condensation transition occurring below the saturated vapour pressure $\mathrm{P}_{0}$.[85] To our knowledge, 
this is the first time that such a "pure" type-IV isotherm is predicted in a nanoporous solid. In previous works, $[39,87]$ we observed that the inclusion of a silanol-type defect could result in type-IV isotherm, but the condensation transition occurred above $\mathrm{P}_{0}$.

We now discuss the effect of the cations on water density. Values for the three systems are shown in Table 3. Porous volumes have been computed using an approach similar to that of the calculation of Connoly surfaces, and is described in detail elsewhere. $[7,88]$ In the case of all-silica and $\mathrm{Na}_{1}$-LTA systems, a negligible amount of water molecules is found in sodalite cages. Thus, the porous volume only takes into account supercages. In the case of $\mathrm{Na}_{1}$-LTA and $\mathrm{Na}_{9}$-LTA, we also compute a porous volume and a density that takes into account the presence of sodium cations (considered as a sphere with a radius being half the $\sigma$ value of sodium, i.e. $1.29 \AA$ ). Water in all-silica LTA is strongly depleted with respect to bulk water, as observed earlier in other siliceous zeolites. $[7,88]$ The presence of only one sodium cation also lowers water density. This is because the presence of one cation is not sufficient to modify strongly water-water interactions (see Section 3.2.1), and because of the volume occupied by the sodium cation. On the other hand, the presence of the nine cations results in a large increase of water density, up to $0.99 \mathrm{~g} . \mathrm{cm}^{-3}$, which is similar to bulk water density. Cations modify water structure in the LTA framework, so that a larger amount of water can be introduced. However, as will be shown in Section 3.2, water structure in $\mathrm{Na}_{9}$-LTA is different from the bulk water.

\subsubsection{On the nature of the condensation transition.}

In order to determine the nature of the condensation transition in all-silica LTA, we have computed the evolution of the grand potential $\Omega$ with pressure. Figure 5a shows the profile of $\Omega_{\mathrm{L}}$ as a function of the water content for various pressures. Below roughly $20 \mathrm{MPa}$, only one stable state exists, that corresponds to an empty LTA zeolite. When pressure is increased, a filled metastable state appears, that is however less stable than the empty state. Around $70 \mathrm{MPa}$, the filled configuration becomes the most stable state. The existence of two stable states is characteristic of a firstorder transition between gas-like and liquid-like phases. The conversion between those two states is possible on a wide range of pressure $(20 \mathrm{MPa} \rightarrow 190 \mathrm{MPa})$, as seen in Figure 5, but the system has to overcome a macroscopic energy barrier. Monte Carlo simulations proceed via microscopic moves. In a finite time MC run, the most stable state may not be reached, and this is why hysteresis can be observed. In the present case, although the equilibrium pressure is approximately $70 \mathrm{MPa}$, intrusion and extrusion occur respectively at $85 \mathrm{MPa}$ and $40 \mathrm{MPa}$ in the simulations.

This is to our knowledge the first time that the Wang-Landau algorithm is used in the context of fluid intrusion in porous solids, and that the existence of a firstorder transition is really demonstrated. We believe that this represents an important progress for the study of adsorption (intrusion) processes, since we are now able to derive thermodynamic quantities that were hardly accessible until now.

\subsection{On the hydrogen bonding of water and the zeolite}

\subsubsection{Structure and orientation dynamics of water.}

To study the interaction of water with the zeolite and add a microscopic insight to the thermodynamic vision of water developed in the previous section, we have first studied the structure and orientation of water confined in the all-silica, $\mathrm{Na}_{1}$ and $\mathrm{Na}_{9}$ LTA zeolites. The arrangement of water molecules in the $\alpha$ cages of the zeolites is 
irregular and reveals none of the features exhibited by gas phase water clusters.[89] In order to probe the nature of the hydrogen bond network of confined water, we have studied the orientation of the molecules around neighbouring water molecules and zeolite oxygen atoms. The key quantities for that task are the $\mathrm{OO}^{\prime}$ distances and $\mathrm{HOO}^{\prime}$ angles (respectively noted $d$ and $\alpha$ in the following, see Figure 6), where $\mathrm{O}$ and $\mathrm{H}$ are atoms from a same water molecule, and $\mathrm{O}^{\prime}$ is a neighbouring oxygen atom. Following the common practice, we used a geometric criterion[90] to characterise hydrogen bonds and study quantitatively the evolution of water structure between the different zeolites: we consider the hydroxyl group $\mathrm{OH}$ of a water molecule hydrogen bonded to an acceptor atom $\mathrm{O}^{\prime}$ if $d \leq 3.5 \AA$ and $\alpha \leq 30^{\circ}$. The limits of the criterion are based on the position of the minima of the $2 \mathrm{D}$ distribution function of liquid water orientation. This criterion is widely used in the literature, as well as a few stricter variants imposing an additional restriction on the $\mathrm{OH}$ distance. Using this criterion, we have studied the evolution of the number of both water-water and water-zeolite hydrogen bonds when the Si:Al ratio of the zeolite is lowered (see Table 4a). First, it can can be seen that the number of water-zeolite hydrogen bonds in the all-silica zeolite is fairly small and that the number of water-water hydrogen bonds is still large, while smaller than for same-density bulk water (around 1.6 per water molecule). The confined water in the hydrophobic, all-silica zeolite can thus be seen as a nanodroplet of liquid water with its hydrogen bonds network largely closed onto itself, and a few $\mathrm{OH}$ groups oriented towards the zeolite. When we move to cationic zeolites, the number of water-zeolite bonds increases while water-water hydrogen bonds diminish; the net result in the total number of hydrogen bonds per water molecule is a slight increase with acidity. As the acidity of the zeolite framework and the number of cations grow, the droplet of confined water "opens up" and forms more hydrogen bonds with the zeolite, at the expense of the water-water hydrogen bonds framework.

To further understand the interaction between the zeolite and the confined water, the classical Monte Carlo simulations were complemented with first-principles molecular dynamics simulations, in order to provide valuable information on the intermolecular and vibrational dynamics of the system as well as to probe its electronic properties (the details of these Car-Parrinello DFT simulations can be found in Section 2.3). Table $4 \mathrm{~b}$ shows the number of water-water and water-zeolite hydrogen bonds counted by the same geometric criterion, for all three zeolites studied. Comparison to Table 4a shows a similar evolutions for both classical models and DFT-based simulations. The absolute number of hydrogen bonds counted is, however, larger in the latter case. This is in keeping with observations that Kohn-Sham DFT liquid water described with the BLYP exchange-correlation function is overstructured at ambient conditions. But the use of molecular dynamics simulations also allows us to get a picture of the dynamics of these hydrogen bonds, in the form of the autocorrelation function of the existence of hydrogen bonds. Figure 7 shows this autocorrelation function for water-zeolite hydrogen bonds in all three zeolites. It can be seen that the $\mathrm{OHO}^{\prime}$ alignments, counted as hydrogen bonds by the geometric criterion, decay very rapidly in the case of all-silica zeolite and $\mathrm{Na}_{1}$-LTA, while the alignments in Nag-LTA subside longer. The hydrogen bond lifetime for each zeolite was obtained by an exponential fit of the 0.05 to $1.5 \mathrm{ps}$ region of the autocorrelation function, where they display monoexponential behaviour. The lifetimes of water-zeolite hydrogen bonds, reported on Figure 7, range from $125 \mathrm{fs}$ to 520 fs and are smaller than the lifetime of water-water hydrogen bonds both in bulk water (1.5 ps, following the same procedure described above) and in confined wa- 
ter (between $1.3 \mathrm{ps}$ and $1.6 \mathrm{ps}$ depending on the number of cations). The very short lifetime of the "geometric H bonds" in both the all-silica zeolite and Na $\mathrm{N}_{1}$ LTA is incompatible with the chemist's view of a hydrogen bond. It is thus plausible that what the geometric criterion counts as hydrogen bonds in these cases are occasional, short-lived $\mathrm{OH} \cdots \mathrm{O}_{\text {zeolite }}$ alignments, corresponding to dangling $\mathrm{OH}$ groups of the confined water. On the other hand, the lifetime of 520 fs observed in Na9-LTA is compatible with weak water-zeolite hydrogen bonds. To characterise these two different situations further, we gain insight from the first-principles dynamics and turn to a electronic characterisation of hydrogen bonds.

\subsubsection{Electronic characterisation of hydrogen bonds: the atomic polar ten-} sor.

By using first-principles molecular dynamics, we can clearly depict the electronic properties of the confined water and thus help understanding its interaction with the confining zeolites. This is needed to characterise hydrogen bonds in a way that will go past the limits of simple geometric criteria. To probe the electronic nature of the hydrogen bonds, we studied the anisotropy of the atomic polar tensor of the hydrogen atoms. In the presence of a hydrogen bond, the hydrogen atom involved exhibits a fairly anisotropic atomic polar tensor. This anisotropy can be characterised by the three singular values of the atomic polar tensor (see Section 2.3). For example, in the case of the water dimer shown in Figure 8, it can be seen that the ellipsoid defined from the singular value decomposition of the APT tensor of the hydrogen atom involved in the hydrogen bonding exhibits a clearly oblong shape, with one singular value much larger than the others and the corresponding singular vector directed along the axis of the hydrogen bond. Physically, the ellipsoid shown in Figure 8 shows the magnitude of dipole moment change upon a small displacement of the hydrogen atoms in the direction of the principal axis. For an hydrogen involved in an $\mathrm{H}$ bond, a displacement parallel to the $\mathrm{H}$ bond can be seen to lead to a much larger change of dipole moment than for a displacement perpendicular to it. This arises from noticeable charge transfer across the $\mathrm{H}$ bond when the hydrogen is moved along the $\mathrm{H}$ bond.

We have thus applied this method to study the strength of both water-water and water-zeolite bonds identified by the geometric criterion; Figure 9 shows the average eigenvalues in each case, as well as for bulk water. It can be seen that the strength for water-water hydrogen bonds in the confined water is fairly similar to that of bulk water: the largest singular value, $\xi_{1}$, is close to $1 e$. Singular values $\xi_{2}$ and $\xi_{3}$, however, are smaller than in the bulk.

The water-zeolite hydrogen bonds, on the other hand, can be seen to be significantly weaker than the water-water bonds. Moreover, we see a gradual increase of $\xi_{1}$ when we go from the all-silica LTA $\left(\left\langle\xi_{1}\right\rangle \simeq 0.36\right)$ to $\mathrm{Na}_{1}$-LTA $\left(\left\langle\xi_{1}\right\rangle \simeq 0.40\right)$ and then Na9-LTA $\left(\left\langle\xi_{1}\right\rangle \simeq 0,77\right)$. Both findings are consistent with the lifetimes reported above, but quantify the nature and strength of these hydrogen bonds in a different perspective: the difference in lifetime, for example, between water-zeolite bonds in Nay-LTA and the bulk water-water hydrogen bond lifetime is markedly more important than the difference in the anisotropy of the atomic polar tensor. The picture they draw for the evolution of water-zeolite interaction with increasing cationic content of the zeolite, however, is the same: while the water confined in the all-silica zeolite can be considered as a droplet forming no hydrogen bonds with the zeolite, but exhibiting a few dangling $\mathrm{OH}$ groups, water confined in the same-structure zeolite with a Si:Al ratio of 1.67 forms relatively weak hydrogen bonds with the oxygen atom of the zeolite 
framework. This difference in the water-zeolite interaction strengthens the explanation of the thermodynamic properties of water condensation in both all-silica and cationic zeolites, as presented in Section 3.1: the hydrophobicity of the internal surface of allsilica zeolites, due to the extreme weakness of the water-zeolite attraction compared to the water-water dispersion energy, leads to intrusion in the liquid phase, while the larger water-zeolite interaction of acidic zeolites leads to adsorption in the gas phase.

The $\mathrm{Na}_{1}-\mathrm{LTA}$, on account of the thermodynamic, dynamic and electronic results shown here, can be seen as a situation midway between the two cases just discussed. Because of the strong electric field created by the presence of an aluminium atom and a cation in the zeolite, water molecules are strongly attracted in the vicinity of the cation, leading to a first water uptake in the gas phase (less than $500 \mathrm{~Pa}$ ). However, in spite of this local attraction, the internal surface is still only weakly attractive for water, as confirmed by the study of the nature of the water-zeolite interaction in $\mathrm{Na}_{1}$-LTA. This leads to the condensation of water at a pressure close to the saturation vapour pressure.

\subsubsection{Infrared spectrum and vibrational spectroscopy of the all-silica zeo- lite.}

To link our simulations to experimentally observable properties, we have also studied the vibrational properties of the all-silica zeolite and the water confined inside its pores. No experimental data is available today on this system, but vibrational spectroscopies have been widely used to investigate the behaviour of water at interfaces. [91, 92] We have calculated the infrared spectrum of both the anhydrous zeolite and the hydrated system. Through the use of the Berry phase approach and maximally localised Wannier functions described in Section 2.3, it was also possible in that latter case to separate the contributions of water and zeolite to the total spectrum. We have first shown that the calculated infrared spectrum of the anhydrous all-silica LTA (Figure 10, lower panel) is in reasonable agreement with the experimental data[93] (Figure 10, upper panel) of the material synthesised by Fyfe et al.[40] The general shape of the spectrum is reproduced, the positions of the absorption bands present relatively large shifts (up to $200 \mathrm{~cm}^{-1}$ ). These are attributed to the fact that the simulation was performed at constant volume, with a unit cell parameter that was not optimised, leading to constraints on the vibrations modes of the zeolite, as well as the dealumination procedure used in Ref. [40], which is known to yield materials with nonnegligible concentrations of defects.[41]

For the hydrated zeolite, we have shown that the presence of water has no significant effect on the infrared spectrum of the zeolite. Moreover, the vibration modes of the zeolite and the confined water are not correlated and the total spectrum of the system matches the sum of the two separate spectra for water and the zeolite, at frequencies higher than $300 \mathrm{~cm}^{-1}$.[29] We have then focused our study on the $\mathrm{OH}$ stretch region of the confined water IR spectrum, shown in Figure 11. It can be seen that, while the $\nu_{\mathrm{OH}}$ peak of bulk water is large and structureless, that of water confined in allsilica LTA exhibits a narrower high-frequency peak on top of a larger band. This peak is attributed to dangling $\mathrm{OH}$ groups, which are not part of the water hydrogen bonds network and are oriented towards the zeolite. Such a peak has been reported for water near a variety of hydrophobic surfaces[94, 95] and can thus be used as a signature of hydrophobicity. As an example of systems where it has been observed, the lower panel of Figure 11 shows the IR SFG (Sum Frequency Generation) spectrum of water near an $\mathrm{H}_{2} \mathrm{O} / \mathrm{CCl}_{4}$ interface, from Scatena et al.[95] In the present case of 
water confined in an all-silica zeolite, we have further confirmed the attribution of this high-frequency $\nu_{\mathrm{OH}}$ peak to dangling $\mathrm{OH}$ groups by studying the correlation between the average stretching frequency of water $\mathrm{OH}$ groups and the percentage of simulation time during which they are involved in water-water hydrogen bonds (according to the geometric criterion). Figure 12 shows that there is a clear correlation between these two quantities: $\mathrm{OH}$ groups with $\nu_{\mathrm{OH}} \geq 2250 \mathrm{~cm}^{-1}$, which compose the high-frequency peak, are markedly less involved in the water-water hydrogen bonds network than the other groups.

\subsection{Dipole of the confined water}

The dipole moment of the water molecules is an important quantity in order to understand the behaviour of confined water and to help assess the quality of the simulation models used. The histogram of the dipole moments of water molecules confined in the $\alpha$ cage of the three zeolites under study are reported in Figure 13, as well as the distribution obtained for a bulk water simulation including 32 water molecules in a periodic unit cell. It can be seen that the distributions for confined water peak around 2.9 D, which is close to the peak value of bulk water at 3.1 D. The self-polarisation of confined water is thus already quite large in these relatively small nanopores; from the point of view of the dipole moment, the confined water is liquid-like. Moreover, Figure 13 and Table 5 reveal that the dependence of the dipole moment on the acidity of the zeolite is very small. The histograms for the all-silica, $\mathrm{Na}_{1}$-LTA and Na9-LTA zeolites have the same peak value, and the only statistically significant difference is that zeolites with more cations present a less symmetric distribution, with a more important high-dipole wing, attributed to water molecules in close contact with the cations.

The relatively small influence of the cationic content of the zeolite is somewhat counterintuitive, as the presence of ionic species in close proximity to the water molecules might be expected to polarise the water molecules significantly. Indeed, calculations performed on a system comprised of a single water molecule close the $\mathrm{Na}^{+}$cation in $\mathrm{Na}_{1}$-LTA have yielded a water dipole moment of $\simeq 2.4 \mathrm{D}$, which represents a large increase $(0.6 \mathrm{D})$ from the gas phase value. These facts lead to the logical conclusion that the dipole moment of the confined water is almost entirely due to the self-polarisation of water, while the influence of both the zeolite and the extraframework cations is faint. Figure 14 presents the histogram of the dipole moments of 15 and 20 water molecules confined in all-silica LTA, which have their maximum at $2.7 \mathrm{D}$ and $2.9 \mathrm{D}$ respectively. This shows that the dependence of the dipole on the water content of the zeolite is more important that the dependence on the number of cations, and that the self-polarisation of water is the main factor determining the dipole in the systems we study here. This conclusion was further strengthened by the analysis of the quadrupole moments of the confined water molecules, presented in Table 5. The quadrupoles are only weakly affected by the changes in the cationic content of the zeolite while they have a more pronounced dependence on the number of water molecules.

\subsection{Partial charges from population analysis and their use as forcefield parameters}

There are many different ways to calculate charges from electronic structure. We have here investigated the atomic charges of zeolite atoms from four different definitions. 
One of our goal was to compare the obtained values from these definitions with charges used in the empirical potentials that were shown to reproduce correct thermodynamics. Two very common methods are the Mulliken population analysis and Hirshfeld population analysis. However, of possible definitions of atomics charges, ESP charges fitted on the electrostatic potential seem the best suited for extracting charges for building an empirical potential. On top of these common atomic charge definitions we have also computed the Born charges obtained as the trace of the APT tensor discussed above. Table 6 shows the resulting charges for atoms of the all-silica zeolite. All these charges give a large spectrum of values. None seem however to agree with the range of empirical charges $(-0.6 e$ to $-0.8 e$ for $\mathrm{O})$ that can correctly describe the hydrophobicity of silicalite-1.[7]

We have also checked the dependance of Mulliken charges on the electronic method used. To this end we selected a few snapshots from the first principle molecular dynamics of empty all-silica zeolite and computed Mulliken charges with a gaussian basis set, using the CP2K program $[96]^{\dagger}$ instead of a plane wave basis set. The resulting charges are $-0.6 e$ for oxygen atoms and $1.2 e$ for silicon atoms, yet again different from other approaches.

Nevertheless, if Mulliken charges are computed for the hydrated all silica zeolite as well, no variation is observed with respect to the empty zeolite. This is consistent with observations made previously in this paper (from the vibrational dynamics analysis for instance) that the coupling between water and the all-silica zeolite is very small. This is also in line with the charges on zeolite atoms independent of filling commonly assumed in empirical forcefields.

Turning now to water, the distribution of Mulliken and Hirshfeld charges are shown in Figure 15. For these two definitions, a decrease of atomic charges is found from an isolated water molecule to bulk liquid water or confined water. Both Mulliken and Hirshfeld population analysis thus give rise to atomic charges that is in contradiction with the increase of water dipole moment. This may be understood by noting that $\mathrm{H}$ bond leads to an increase of electron density in the intermolecular region between $\mathrm{O}$ and $\mathrm{H}$. Instead of an increase of molecular polarisation, the Mulliken and Hirsfeld population analysis attribute some of that density to the partner hydrogen of a donating oxygen, leading to a decrease of absolute values of charges arising from this apparent intermolecular charge transfer.

On the contrary, the Born charges (see Figure 16) show an increase of atomic charges upon going from an isolated molecule to a confined water molecule and to a bulk water molecule. This increase of Born atomic charges is furthermore in near quantitative agreement with the dipole moment variation: assigning these charges to hydrogen and oxygen atoms, we find dipole moments of $1.4 \mathrm{D}, 2.77 \mathrm{D}$ and $3.2 \mathrm{D}$ for an isolated molecule, a confined molecule and a bulk water molecule, respectively. These charges also appear to be in the range of partial charges found in empirical forcefields for liquid water.

From considerations of infrared intensities, [82] it is possible to define an alternative to the Born charge from the APT tensor by

$$
\xi^{2}=\frac{\xi_{1}^{2}+\xi_{2}^{2}+\xi_{3}^{2}}{3}
$$

\footnotetext{
${ }^{\dagger}$ DFT calculations were performed with the BLYP functional and Goedecker-Tetter-Hutter pseudopotentials in the GPW approach, with DZVP basis set and a cutoff of 300 Ry for the description of the electronic density in plane waves.
} 
where $\xi_{1}, \xi_{2}$ and $\xi_{3}$ are the singular values of the APT tensor as discussed above and $\xi$ has thus the dimension of a charge. These charges are found to be slightly lower that the Born charges $\left(\xi_{H}=0.39 e\right.$ for confined water and $0.54 e$ for bulk liquid) but show the same trend, with an increase of molecular polarisation from confined to bulk water.

APT derived charges and in particular Born charges appear as interesting candidates to define meaningful atomic partial charges, at least for molecular systems. As mentioned before the Born charges of the zeolite atoms appear however too large with respect to the range of charges used in classical forcefields.

\section{Concluding remarks}

The use of both classical GCMC and first-principles CPMD simulations has allowed to describe in some details the behaviour of a nanodroplet of water confined in the supercage of LTA zeolites. Each method has its (known) strengths and drawbacks, and provides information in a physical chemistry domain where the other does not. Whenever the same kind of information can be obtained by both techniques, the agreement was found satisfactory. This was the case for instance for the number of hydrogen bonds between water molecules and the framework atoms as well as between water molecules themselves. Work is in progress to get a better insight into the hydrogen bond reorientation dynamics of water in such confined systems.[97]

The all-silica version of LTA as well as two model systems with 1 and 9 sodium cations were chosen for this study. From the thermodynamic point of view, we have identified the all-silica LTA zeolite as hydrophobic, and the cationic Nag-LTA zeolites as hydrophilic. The condensation transition in the first case was demonstrated to be of first order. The adsorption isotherm in the case $\mathrm{Na}_{1}$-LTA and Nag-LTA was of Type IV and I respectively. Furthermore, the in-depth analysis of the dynamic and electronic properties of water showed that water in the hydrophobic zeolite behaves as a nanodroplet trying to close its hydrogen bonds onto itself, with a few shortlived dangling $\mathrm{OH}$ groups, while water in the hydrophilic zeolites opens up to form weak hydrogen bonds with the oxygen atoms of the framework. We conclude that a thoughtful combination of methods, in which each technique is used for what it was designed for, can provide a nice insight into the physics and chemistry of such a complex interfacial system as water confined in zeolite nanopores.

\section{Acknowledgments}

This work was supported by the French Agence Nationale de la Recherche, under contracts \# JC05_51932 and BLANC06-3_144027. We acknowledge the Institut du Développement et des Ressources en Informatique Scientifique (IDRIS) and ENSCP for computer time allocation. We thank Isabelle Demachy, Florent Calvo and Nicolas Desbiens for fruitful discussions. 


\section{References}

[1] T. Ohba, H. Kanoh, and K. Kaneko, J. Am. Chem. Soc., 2004, 126, 1560-1562.

[2] S. Devautour, S. A. Abdoulaye, J.-C. Giuntini, and F. Henn, J. Phys. Chem. B, 2001, 105, 9297-9301.

[3] P. Demontis and G. B. Suffritti, Chem. Rev., 1997, 97, 2845-2878.

[4] B. Boddenberg, G. U. Rakhmatkariev, S. Hufnagel, and Z. Salimov, Phys. Chem. Chem. Phys., 2002, 4, 4172-4180.

[5] J.-C. Moïse, J.-P. Bellat, and A. Méthivier, Microporous Mesoporous Mater., 2001, 43, 91-101.

[6] N. Giovambattista, P. J. Rossky, and P. G. Debenedetti, Phys. Rev. E, 2006, 73, 041604 .

[7] N. Desbiens, A. Boutin, and I. Demachy, J. Phys. Chem. B, 2005, 109, 2407124076 .

[8] C. E. Ramachandran, S. Chempath, L. J. Broadbelt, and R. Q. Snurr, Microporous Mesoporous Mater., 2006, 90, 293-298.

[9] W. Kauzmann, Adv. Prot. Chem., 1959, 14, 1-63.

[10] K. A. Dill, Biochemistry, 1990, 29, 7133-7155.

[11] D. Chandler, Nature, 2002, 417, 491-491.

[12] C. L. Brooks, J. N. Onuchic, and D. J. Wales, Science, 2001, 293, 612-613.

[13] C. M. Dobson, A. Sali, and M. Karplus, Angew. Chem. Int. Ed., 1998, 37, 868-893.

[14] O. Beckstein and M. S. P. Sansom, Proc. Natl. Acad. Sci. USA, 2003, 100, 70637068 .

[15] G. Hummer, J.-C. Rasaiah, and J. P. Noworyta, Nature, 2001, 414, 188-190.

[16] J. Dzubiella and J.-P. Hansen, J. Chem. Phys., 2005, 122, 234706.

[17] A. Otten and S. Herminghaus, Langmuir, 2004, 20, 2405-2408.

[18] J. W. G. Tyrell and P. Attard, Phys. Rev. Lett., 2001, 87, 176104.

[19] R. Steitz, T. Gutberlet, T. Hauss, B. Klösgen, R. Krastev, S. Schemmel, A. C. Simonsen, and G. H. Findenegg, Langmuir, 2003, 19, 2409-2418.

[20] R. A. Jackson and C. R. A. Catlow, Mol. Simulat., 1988, 1, 207-224.

[21] M. J. Sanders, M. Leslie, and C. R. A. Catlow, J. Chem. Soc. Chem. Comm., 1984, pp. 1271-1273.

[22] N. H. de Leeuw and S. C. Parker, J. Am. Ceram. Soc., 1999, 82, 3209-3216.

[23] F. Manon-Higgins, N. H. de Leeuw, and S. C. Parker, J. Mater. Chem., 2002, 12, $124-131$. 
[24] A. R. Ruiz-Salvador, D. W. Lewis, J. Rubayo-Soneira, G. Rodriguez-Fuentes, L. R. Sierra, and C. R. A. Catlow, J. Phys. Chem. B, 1998, 100, 6722-6730.

[25] E. Fois, A. Gamba, G. Tabacchi, S. Quartieri, and G. Vezzalini, Phys. Chem. Chem. Phys., 2001, 3, 4158-4163.

[26] E. Fois, A. Gamba, G. Tabacchi, S. Quartieri, and G. Vezzalini, J. Phys. Chem. $B, 2001, \mathbf{1 0 5}, 3012-3016$.

[27] E. Fois, A. Gamba, and A. Tilocca, J. Phys. Chem. B, 2002, 106, 4806-4812.

[28] C. Ceriani, A. Gamba, G. Tabacchi, O. Ferro, S. Quartieri, and G. Vezzalini, Am. Mineral., 2004, 89, 102-109.

[29] F.-X. Coudert, R. Vuilleumier, and A. Boutin, ChemPhysChem, 2006, 7, 24642467.

[30] C. Bussai, S. Fritzsche, R. Haberlandt, and S. Hannongbua, J. Phys. Chem. B, 2003, 107, 12444-12450.

[31] K. S. Smirnov and D. Bougeard, Chem. Phys., 2003, 292, 53-70.

[32] C. Beauvais, A. Boutin, and A. H. Fuchs, ChemPhysChem, 2004, 5, 1791-1793.

[33] G. Maurin, R. G. Bell, S. Devautour, F. Henn, and J.-C. Giuntini, J. Phys. Chem. $B, 2004,108,3739-3745$.

[34] K. Shirono, A. Endo, and H. Daiguji, J. Phys. Chem. B, 2005, 109, 3446-3453.

[35] N. A. Ramsahye and R. G. Bell, J. Phys. Chem. B, 2005, 109, 4728-4747.

[36] K. Shirono and H. Daiguji, Chem. Phys. Lett., 2006, 417, 251-255.

[37] N. Desbiens, I. Demachy, A. H. Fuchs, H. Kirsch-Rodeschini, M. Soulard, and J. Patarin, Angew. Chem. Int. Ed., 2005, 44, 5310-5313.

[38] D. Bougeard and K. S. Smirnov, Phys. Chem. Chem. Phys., 2007, 9, 226.

[39] M. Trzpit, M. Soulard, J. Patarin, N. Desbiens, F. Cailliez, A. Boutin, I. Demachy, and A. H. Fuchs, Langmuir, 2007, 23, 10131-10139.

[40] C. A. Fyfe, G. J. Kennedy, G. T. Kokotailo, and C. T. DeSchutter, J. Chem. Soc., Chem. Commun., 1984, pp. 1093-1094.

[41] D. Siantar, W. Millman, and J. Fripiat, Zeolites, 1995, 15, 556-560.

[42] A. Corma, F. Rey, J. Rius, M. J. Sabater, and S. Valencia, Nature, 2004, 431, $287-290$.

[43] P. Caullet, J. L. Paillaud, A. Simon-Masseron, M. Soulard, and J. Patarin, C. R. Chimie, 2005, 8, 245-266.

[44] J. Patarin, private communication, 2006.

[45] C. Baerlocher, W. M. Meier, and D. H. Olson, Atlas of zeolite framework types, 2001. 
[46] W. Loewenstein, Am. Mineral., 1954, 39, 92-96.

[47] R. Y. Yanagida, A. A. Amaro, and K. Seff, J. Phys. Chem., 1973, 77, 805-809.

[48] A. H. Fuchs and A. K. Cheetham, J. Phys. Chem. B, 2001, 105, 7375-7383.

[49] N. Desbiens, I. Demachy, A. H. Fuchs, H. Kirsch-Rodeschini, M. Soulard, and J. Patarin, Angew. Chem. Int. Edit., 2005, 44, 5310-5313.

[50] A. Di Lella, N. Desbiens, A. Boutin, I. Demachy, P. Ungerer, J.-P. Bellat, and A. H. Fuchs, Phys. Chem. Chem. Phys., 2006, 8, 5396-5406.

[51] C. Beauvais, X. Guerrault, F.-X. Coudert, A. Boutin, and A. H. Fuchs, J. Phys. Chem. B, 2004, 108, 399-404.

[52] E. Jaramillo and S. M. Auerbach, J. Phys. Chem. B, 1999, 103, 9589-9594.

[53] W. L. Jorgensen and J. D. Madura, Mol. Phys., 1985, 56, 1381-1392.

[54] D. Nicholson and N. G. Parsonage, Computer Simulation and the Statistical Mechanics of Adsorption, Academic Press: New York, 1982.

[55] D. Frenkel and B. Smit, Understanding molecular simulation: from algorithms to applications, Academic Press: London, 2002.

[56] A. D. Mackie, B. Tavitian, A. Boutin, and A. H. Fuchs, Mol. Simulat., 1997, 19, $1-15$.

[57] R. F. Cracknell, D. Nicholson, N. G. Parsonage, and H. Evans, Mol. Phys., 1990, 71, 931-943.

[58] V. Lachet, A. Boutin, B. Tavitian, and A. H. Fuchs, J. Phys. Chem. B, 1998, 102, 9224-9233.

[59] F. Wang and D. Landau, Phys. Rev. E, 2001, 64, 056101.

[60] F. Wang and D. Landau, Phys. Rev. Lett., 2001, 86, 2050-2053.

[61] M. Shell, P. Debenedetti, and A. Panagiotopoulos, Phys. Rev. E, 2002, 66, 056703.

[62] P. Poulain, F. Calvo, R. Antoine, M. Broyer, and P. Dugourd, Phys. Rev. E, 2006, 73, 056704 .

[63] N. Rathore and J. de Pablo, Abstr. Am. Chem. Soc., 2002, 224, U485-U485.

[64] E. Kim, R. Faller, Q. Yan, N. Abbott, and J. de Pablo, J. Chem. Phys., 2002, 117, 7781-7787.

[65] N. Rathore, Q. Yan, and J. de Pablo, J. Chem. Phys., 2004, 120, 5781-5788.

[66] M. Chopra, M. Müller, and J. de Pablo, J. Chem. Phys., 2006, 124, 134102.

[67] C. Zhou and R. Bhatt, Phys. Rev. E, 2005, 72, 025701.

[68] R. Car and M. Parrinello, Phys. Rev. Lett., 1985, 55, 2471. 
[69] CPMD, Copyright IBM Corp. 1990-2006, Copyright MPI für Festkörperforschung Stuttgart 1997-2001.

[70] A. D. Becke, Phys. Rev. A, 1988, 38, 3098.

[71] C. Lee, W. Yang, and R. G. Parr, Phys. Rev. B, 1988, 37, 785.

[72] S. Nosé, Mol. Phys., 1984, 52, 255-268.

[73] W. G. Hoover, Phys. Rev. A, 1985, 31, 1695-1697.

[74] R. Resta, Phys. Rev. Lett., 1998, 80, 1800.

[75] P. Silvestrelli and M. Parrinello, J. Chem. Phys., 1999, 111, 3572.

[76] R. Ramrez, T. Lopez-Ciudad, P. Kumar, and D. Marx, J. Chem. Phys., 2004, 121, 3973-3983.

[77] M.-P. Gaigeot and M. Sprik, J. Phys. Chem. B, 2003, 107, 10344.

[78] M. Gaigeot, R. Vuilleumier, M. Sprik, and D. Borgis, J. Chem. Theory Comput., $2005,1,772$.

[79] N. Marzari and D. Vanderbilt, Phys. Rev. B, 1997, 56, 12847.

[80] M. Sharma, R. Resta, and R. Car, Phys. Rev. Lett., 2005, 95, 187401.

[81] R. Iftimie and M. E. Tuckerman, J. Chem. Phys., 2005, 122(21), 214508.

[82] R. Vuilleumier, Liquid water dynamical partial charges and infrared intensities, http://hal.archives-ouvertes.fr/hal-00267645/en/, to be submitted.

[83] X. Gonze, Phys. Rev. B, 1997, 55, 10337-10354.

[84] A. Putrino, D. Sebastiani, and M. Parrinello, J. Chem. Phys., 2000, 113, 71027109 .

[85] F. Rouquerol, J. Rouquerol, and K. Sing, Adsorption by Powders and Porous Solids, Academic Press: New York, 1999.

[86] V. Eroshenko, R.-C. Regis, M. Soulard, and J. Patarin, J. Am. Chem. Soc., 2001, 123, 8129-8130.

[87] F. Cailliez, G. Stirnemann, A. Boutin, I. Demachy, and A. H. Fuchs, J. Phys. Chem. C, 2008, submitted.

[88] F. Cailliez, N. Desbiens, I. Demachy, M. Trzpit, M. Soulard, J. Patarin, and A. H. Fuchs, Proceedings of 4th International FEZA Conference, 2008, submitted.

[89] The Cambridge Cluster Database, D. J. Wales, J. P. K. Doye, A. Dullweber, M. P. Hodges, F. Y. Naumkin, F. Calvo, J. Hernández-Rojas and T. F. Middleton, http://www-wales.ch.cam.ac.uk/CCD.html.

[90] A. Luzar and D. Chandler, Nature, 1996, 379, 55-57.

[91] C. Bain, J. Chem. Soc., Faraday Trans., 1995, 91, 1281-1296. 
[92] Y. R. Shen and V. Ostroverkhov, Chem. Rev., 2006, 106, 1140-1154.

[93] Y. Huang and Z. Jiang, Microporous Mater., 1997, 12, 341-345.

[94] P. B. Miranda and Y. R. Shen, J. Phys. Chem. B, 1999, 103, 3292-3307.

[95] L. Scatena, M. Brown, and G. Richmond, Science, 2001, 292, 908-912.

[96] The CP2K developers group, http://cp2k.berlios.de/, 2007.

[97] D. Laage, F.-X. Coudert and A. Boutin, to be published. 
Table 1: Forcefield parameters used for modelling repulsion and dispersion interactions in Monte Carlo simulations.

\begin{tabular}{lll}
\hline Interaction & Parameter & Value \\
\hline $\mathrm{Na}-\mathrm{Na}$ & $\sigma$ & $2.584 \AA$ \\
& $\epsilon$ & $50.27 \mathrm{~K}$ \\
$\mathrm{O}_{\text {water }}-\mathrm{O}_{\text {water }}$ & $\sigma$ & $3.154 \AA$ \\
& $\epsilon$ & $78.03 \mathrm{~K}$ \\
$\mathrm{O}_{\text {zeolite }}-\mathrm{O}_{\text {zeolite }}$ & $\sigma$ & $3.000 \AA$ \\
$\mathrm{Na}^{-} \mathrm{O}_{\text {zeolite }}$ & $\epsilon$ & $93.53 \mathrm{~K}$ \\
& $\alpha$ & $61.1 \times 10^{6} \mathrm{~K}$ \\
& $\beta$ & $4.05 \AA^{-1}$ \\
& $\gamma$ & $76.52 \times 10^{4} \mathrm{~K} . \AA^{6}$ \\
\hline
\end{tabular}

Table 2: Partial charges borne by atoms in the Monte Carlo simulations.

\begin{tabular}{ll}
\hline Molecule & Charges $(e)$ \\
\hline TIP4P water & $\mathrm{qO}_{\mathrm{O}}=-1.04$ \\
& $\mathrm{q}_{\mathrm{H}}=+0.52$ \\
all-silica LTA framework & $\mathrm{q}_{\mathrm{O}}=-0.7$ \\
& $\mathrm{q}_{\mathrm{Si}}=+1.4$ \\
$\mathrm{Na}_{1}$-LTA framework & $\mathrm{q}_{\mathrm{O}}=-0.7125$ \\
& $\mathrm{q}_{\mathrm{T}}=+1.3833$ \\
$\mathrm{Na}_{9}$-LTA framework & $\mathrm{q}_{\mathrm{O}}=-0.8125$ \\
& $\mathrm{q}_{\mathrm{T}}=+1.25$ \\
$\mathrm{Na}^{+}$ & $\mathrm{q}_{\mathrm{Na}}=+1$ \\
\hline
\end{tabular}

Table 3: Filling of LTA at saturation, porous volume, and computed density of water. The porous volumes and densities shown in brackets take into account the excluded volume due to sodium cations.

\begin{tabular}{|c|c|c|c|}
\hline System & N/u.c. & $V_{\mathrm{P}} /$ u.c. $\left(\AA^{3}\right)$ & $\rho_{\text {conf }}\left(\right.$ g.cm $\left.{ }^{-3}\right)$ \\
\hline LTA all-silica & 21 & 754 & 0.83 \\
\hline $\mathrm{Na}_{1}$-LTA & 20 & $754(745)$ & $0.79(0.80)$ \\
\hline $\mathrm{Na}_{9}$-LTA & 30 & $983(901)$ & $0.91(0.99)$ \\
\hline
\end{tabular}


Table 4: Number of hydrogen bonds among the water molecules confined inside the $\alpha$ cage of LTA zeolites with different numbers of cations, using a geometric criterion (see text), from Monte Carlo simulations (upper table) and first-principles molecular dynamics (lower table). For each zeolite, the number of water-water hydrogen bonds and water-zeolite hydrogen bonds is indicated at well as the ratios per water molecule.

(a) From Monte Carlo simulations

\begin{tabular}{|c|c|c|c|c|c|}
\hline \multirow[t]{2}{*}{ zeolite } & \multicolumn{2}{|c|}{ water-water $\mathrm{H}$ bonds } & \multicolumn{2}{|c|}{ water-zeolite $\mathrm{H}$ bonds } & \multirow{2}{*}{$\begin{array}{l}\text { all } \mathrm{H} \text { bonds } \\
\text { per } \mathrm{H}_{2} \mathrm{O}\end{array}$} \\
\hline & total & per $\mathrm{H}_{2} \mathrm{O}$ & total & per $\mathrm{H}_{2} \mathrm{O}$ & \\
\hline all-silica & 25.6 & 1.35 & 3.9 & 0.20 & 1.55 \\
\hline one cation & 23.2 & 1.22 & 6.0 & 0.32 & 1.54 \\
\hline nine cations & 17.6 & 0.93 & 13.0 & 0.68 & 1.61 \\
\hline
\end{tabular}

(b) From first-principles molecular dynamics

\begin{tabular}{|c|c|c|c|c|c|}
\hline \multirow[t]{2}{*}{ zeolite } & \multicolumn{2}{|c|}{ water-water $\mathrm{H}$ bonds } & \multicolumn{2}{|c|}{ water-zeolite $\mathrm{H}$ bonds } & \multirow{2}{*}{$\begin{array}{l}\text { all } \mathrm{H} \text { bonds } \\
\text { per } \mathrm{H}_{2} \mathrm{O}\end{array}$} \\
\hline & total & per $\mathrm{H}_{2} \mathrm{O}$ & total & per $\mathrm{H}_{2} \mathrm{O}$ & \\
\hline all-silica & 30.8 & 1.54 & 4.1 & 0.21 & 1.75 \\
\hline one cation & 27.2 & 1.43 & 7.1 & 0.37 & 1.80 \\
\hline nine cations & 22.9 & 1.21 & 14.0 & 0.74 & 1.95 \\
\hline
\end{tabular}

Table 5: Dipole moment and quadrupole eigenvalues of water confined in different LTA zeolites. The values for bulk water, ice and an isolated water molecule are also shown for comparison purposes.

\begin{tabular}{rllll}
\hline system & dipole $(\mathrm{D})$ & \multicolumn{3}{c}{ quadrupole eigenvalues $\left(\mathrm{esu}^{\mathrm{c}} \mathrm{cm}^{2}\right)$} \\
\hline $15 \mathrm{H}_{2} \mathrm{O}$ in all-silica LTA & 2.79 & -3.04 & -0.17 & 3.21 \\
$20 \mathrm{H}_{2} \mathrm{O}$ in all-silica LTA & 2.83 & -3.18 & -0.14 & 3.32 \\
$20 \mathrm{H}_{2} \mathrm{O}$ in Na -LTA & 2.83 & -3.14 & -0.12 & 3.27 \\
$20 \mathrm{H}_{2} \mathrm{O}$ in Nag-LTA & 2.88 & -3.06 & -0.12 & 3.18 \\
$1 \mathrm{H}_{2} \mathrm{O}$ in Na $\mathrm{Na}_{1}$-LTA & 2.38 & -2.57 & -0.26 & 2.82 \\
\hline isolated water molecule & 1.84 & -2.47 & -0.10 & 2.57 \\
bulk liquid water & 3.0 & -3.19 & -0.19 & 3.38 \\
ice & 3.44 & -3.69 & -0.12 & 3.81 \\
\hline
\end{tabular}

Table 6: Average partial charges on the oxygen and silicon atoms of the all-silica zeolite, depending on the analysis method used: Mulliken, Hirshfeld, ESP and Born (see Section 3.4).

\begin{tabular}{rll}
\hline method & oxygen & silicon \\
\hline Mulliken & -1.007 & 2.175 \\
Hirshfeld & -0.293 & 0.586 \\
ESP & -1.901 & 3.801 \\
Born & -1.557 & 3.114 \\
\hline
\end{tabular}




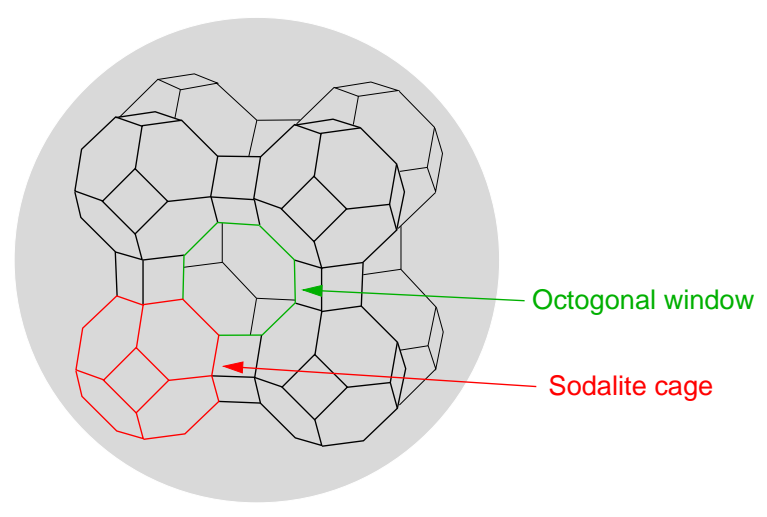

Figure 1: Schematic view of the LTA structure showing an $\alpha$ cage (center) with its eight surrounding sodaliate cages. $\alpha$ cages are linked to one another by octogonal windows.
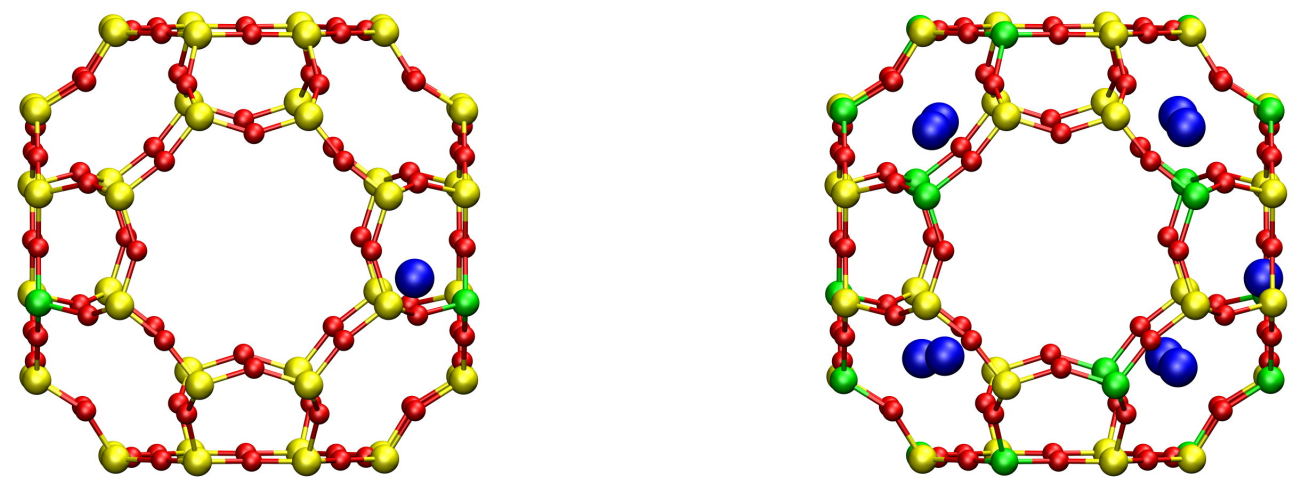

Figure 2: Left: Structure of the $\mathrm{Na}_{1}$-LTA zeolite, containing one aluminium atom (in green) and one extraframework $\mathrm{Na}^{+}$cation (in blue). The cation is represented in its most stable cristallographic site, known as Na(3).[47] Right: Structure of Nag-LTA zeolite with nine aluminium atoms and $9 \mathrm{Na}^{+}$cations. Eight cations are in sites $\mathrm{Na}(1)$, and one is in a site $\mathrm{Na}(3)$. 


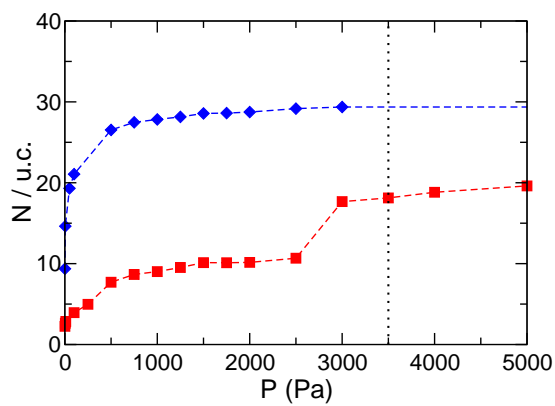

(a)

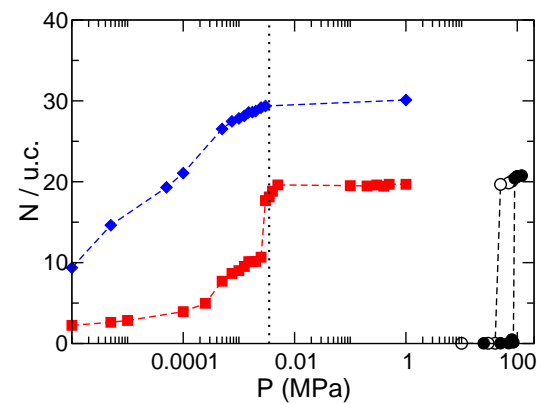

(b)

Figure 3: (a) Computed adsorption isotherms of water in $\mathrm{Na}_{1}$-LTA (red squares) and $\mathrm{Na}_{9}$-LTA (blue diamonds) at $300 \mathrm{~K}$; (b) Intrusion (filled circles) and extrusion (open circles) isotherm of water in all-silica LTA (in black). Adsorption curves for $\mathrm{Na}_{1}$-LTA and $\mathrm{Na}_{9}$-LTA are also reported for comparison sake. A vertical dotted line indicates $P_{0}$, the water saturation vapour pressure at $300 \mathrm{~K}$.

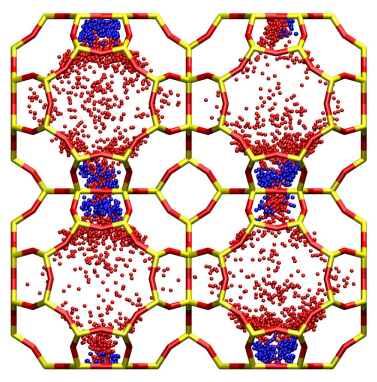

(a)

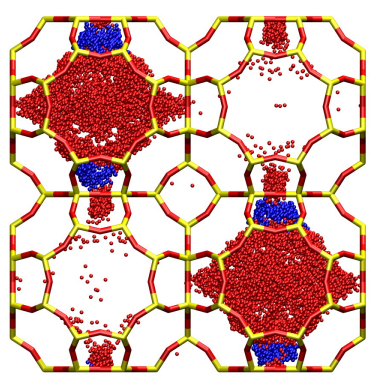

(b)

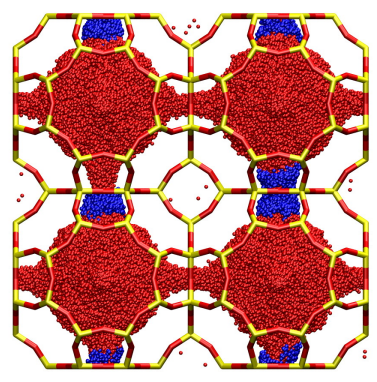

(c)

Figure 4: Water filling of $\mathrm{Na}_{1}$-LTA zeolite from GCMC simulations. Each dot represents the position of molecules (water in red and sodium in blue) accumulated over the simulation run (after convergence has been reached) at $250 \mathrm{~Pa}$ (a), $1000 \mathrm{~Pa}$ (b), and $3000 \mathrm{~Pa} \mathrm{(c).} \mathrm{Zeolite} \mathrm{framework} \mathrm{is} \mathrm{shown} \mathrm{in} \mathrm{sticks} \mathrm{(oxygen} \mathrm{atoms} \mathrm{in} \mathrm{red} \mathrm{and} \mathrm{silicon}$ atoms in yellow). For the sake of clarity, only 4 unit cells are shown. 


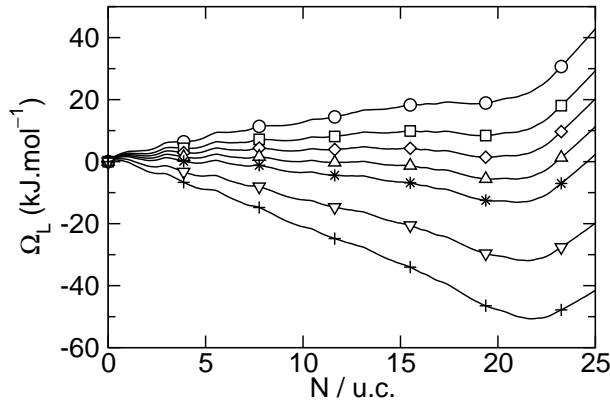

(a)

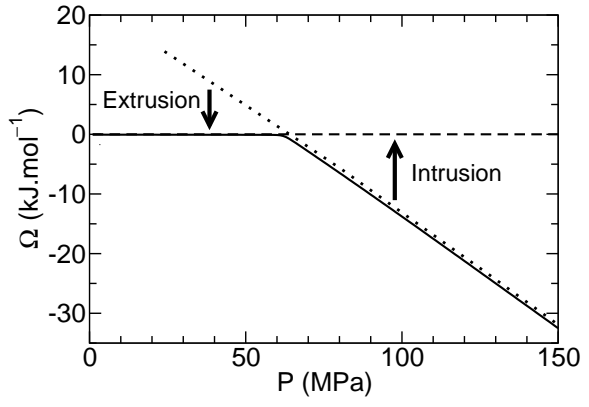

(b)

Figure 5: (a) Landau free energy profile as a function of water content of all-silica LTA at $10 \mathrm{MPa}$ (circles), $40 \mathrm{MPa}$ (squares), $60 \mathrm{MPa}$ (diamonds), $80 \mathrm{MPa}$ (up triangles), $100 \mathrm{MPa}$ (stars), $150 \mathrm{MPa}$ (down triangles), and $200 \mathrm{MPa}$ (plus symbols). (b) Variation of the grand potential $\Omega$ at equilibrium with pressure (thin plain line). The dashed and dotted lines represent the value of $\Omega$ for the metastable empty and filled states respectively.

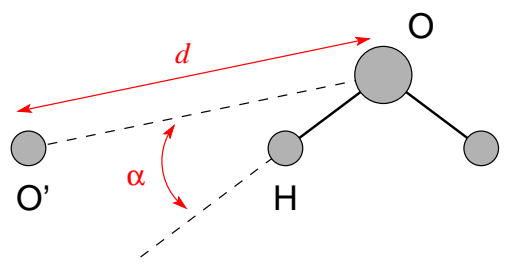

Figure 6: Definition of the parameters $d$ and $\alpha$ used to characterise the water orientation and on which the geometric criterion for hydrogen bonds is based.

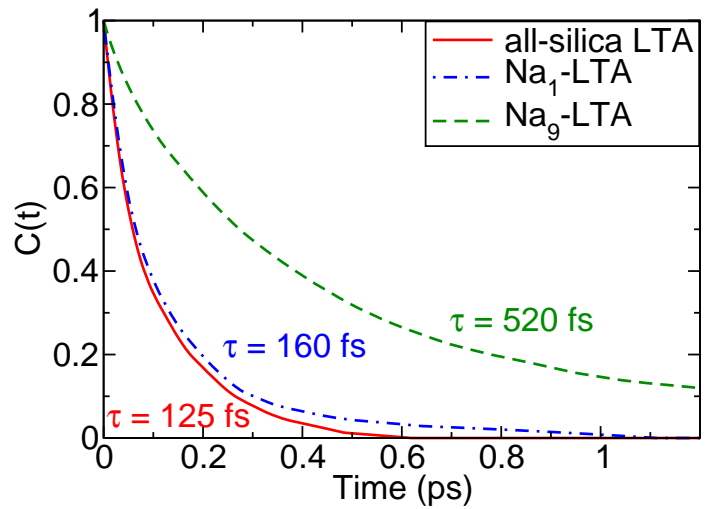

Figure 7: Autocorrelation function $C(t)$ of the existence of water-zeolite hydrogen bonds, as defined per the geometric criterion described in the text, for water confined in different zeolites of identical framework type: all-silica (red), one cation (blue) and nine cations (red). The lifetime of the bond, indicated on each curve, is obtained by an exponential fit of the $0.05-1.5$ ps region of the autocorrelation function. 


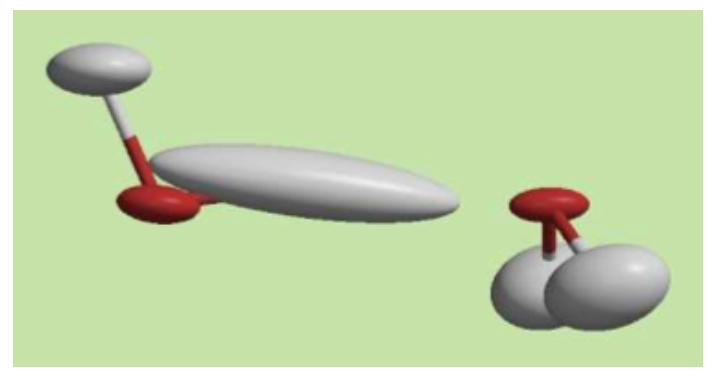

Figure 8: Graphical representation of the Atomic Polar Tensor (APT) of atoms in a water dimer. The ellipsoids are drawn from the singular value decompositions of the APT tensors. They describe the magnitude of dipole moment changes upon displacement of the atoms in the principal directions of the ellipsoids. The lengths of the principal axis of these ellipsoids are thus the singular values of the APT tensors. It can be noted that the hydrogen involved in the hydrogen bond exhibits a significant anisotropy of the APT tensor.

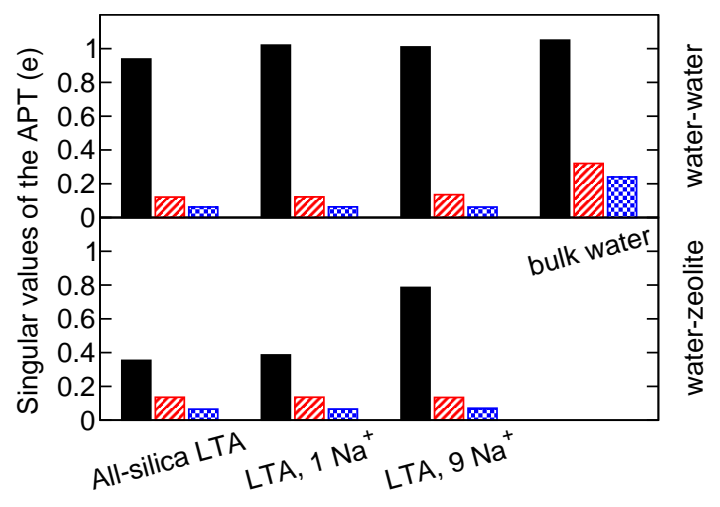

Figure 9: Average singular values of the symmetric of the atomic polar tensor (see Section 2.3) for hydrogen atoms of water confined in the three zeolites (all-silica, one cation and nine cations): $\xi_{1}$ in black, $\xi_{2}$ in red and $\xi_{3}$ is blue. The unit of the singular values is the atomic unit of charge, $e$. The upper panel features the singular values for hydrogen atoms involved in water-water hydrogen bonds; the values for bulk water are indicated for comparison. The lower panel features singular values for hydrogen atoms oriented towards the zeolite. 


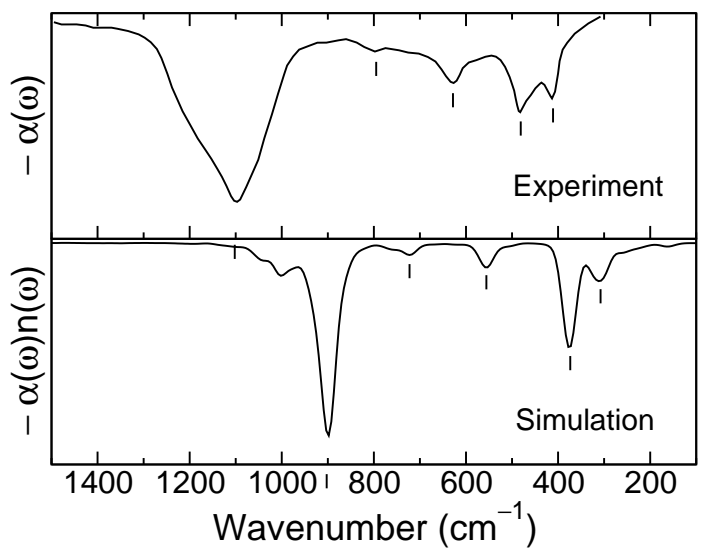

Figure 10: Infrared spectrum of the dehydrated, LTA zeolite: computed from firstprinciples molecular dynamics of the all-silica zeolite (lower panel), compared to experimental data from a reportedly all-silica zeolite[40] (upper panel). 

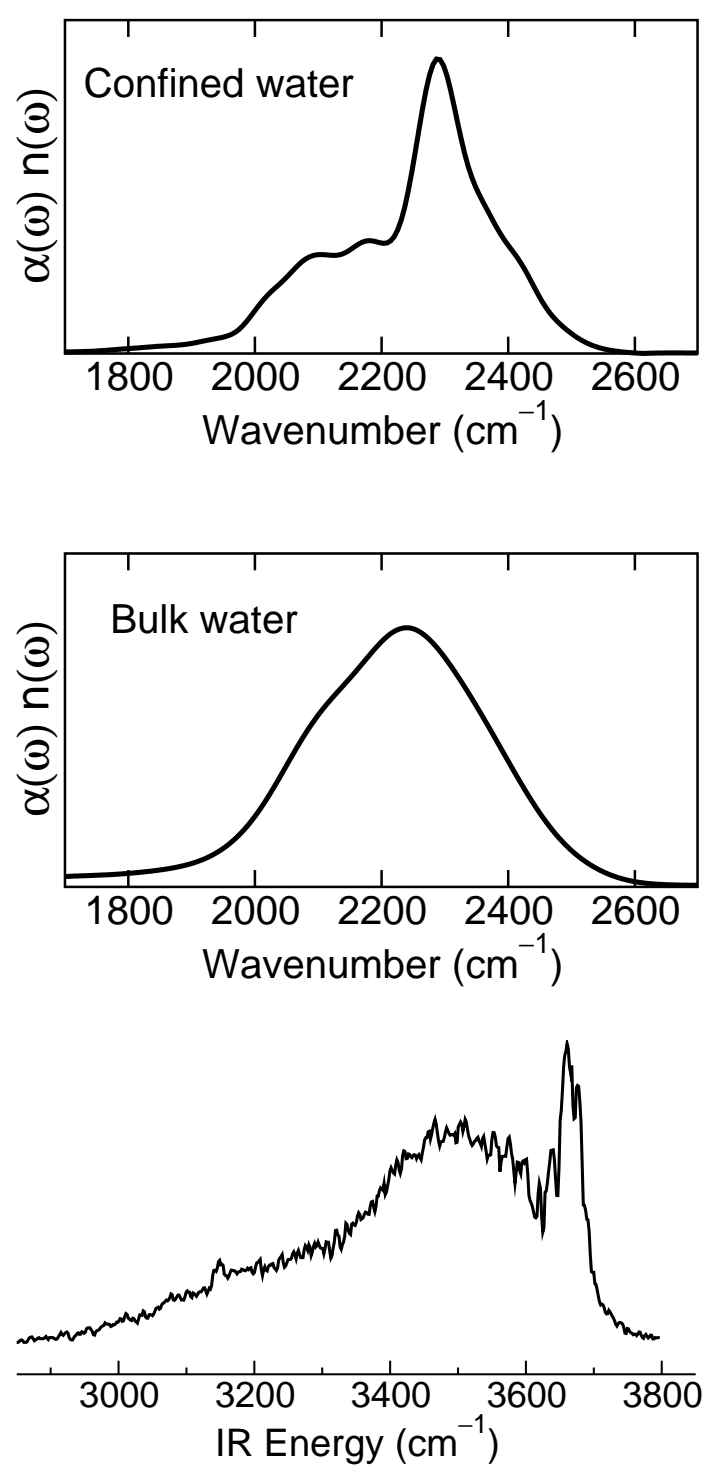

Figure 11: OH stretch band of the infrared spectrum of 20 water molecules confined in all-silica LTA zeolite (upper panel) and 32 bulk water molecules (middle panel). The lower panel shows the Vis-IR SFG spectrum of water near an $\mathrm{H}_{2} \mathrm{O} / \mathrm{CCl}_{4}$ interface, from Scatena et al.[95], exhibiting a high-frequency peak considered as a signature of hydrophobicity. The simulated systems (upper and middle panel) are deuterated, hence the difference in vibration frequency. 


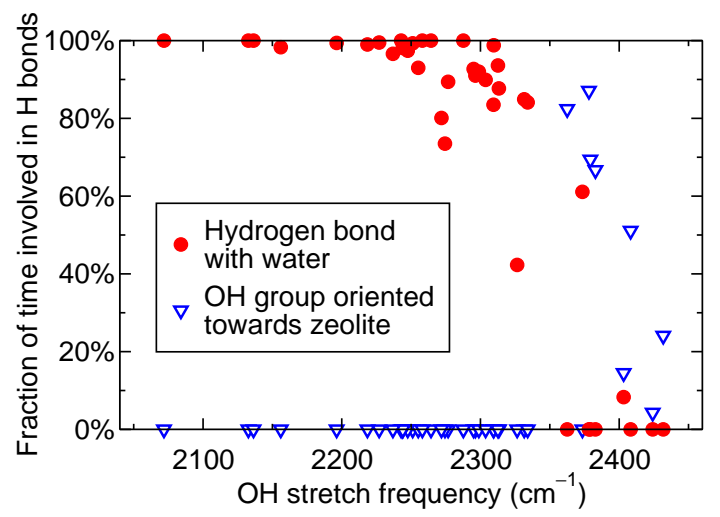

Figure 12: Correlation between the average stretching frequency (horizontal axis) of a water $\mathrm{OH}$ group confined in an all-silica LTA and the percentage of simulation time during which this $\mathrm{OH}$ group is involved in water-water hydrogen bonds (vertical axis, red circles) and is oriented towards the zeolite (vertical axis, blue triangles).

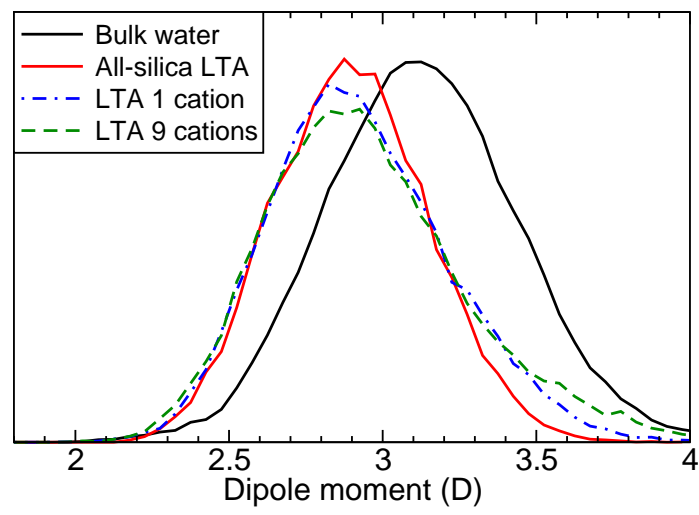

Figure 13: Distribution of the dipole moment of water molecules in confined in the all-silica LTA (red), $\mathrm{Na}_{1}$-LTA (blue) and Nag-LTA (green). The distribution of dipole moments in bulk water is drawn in black. 


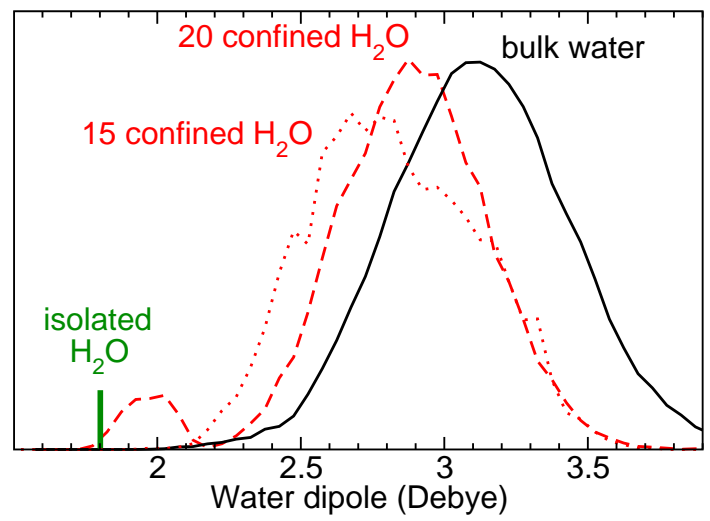

Figure 14: Distribution of the dipole moment of water molecules in bulk water (black, full line), of 20 water molecules confined in an all-silica LTA zeolite (red, dashed line) and of 15 water molecules inside the all-silica zeolite (red, dotted line). The dipole moment of a water molecule in gas phase is also presented (green line).

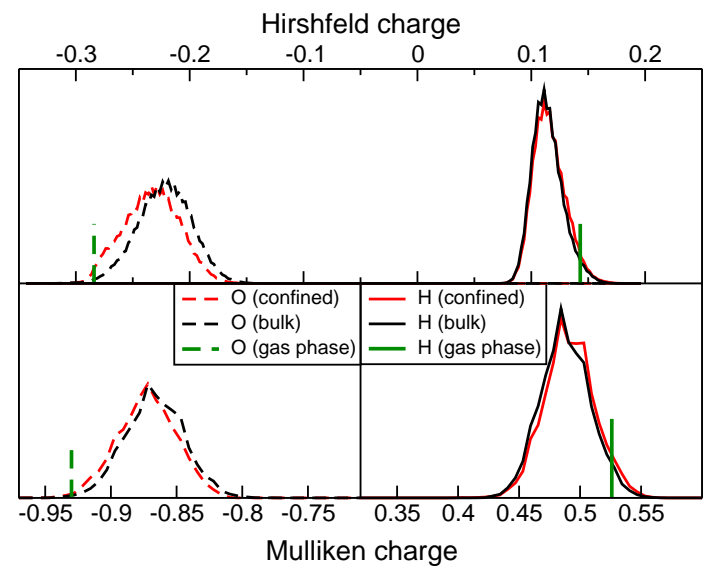

Figure 15: Distribution of partial charges of the water oxygen and hydrogen atoms according to the Mulliken and Hirshfeld population analysis methods. The distributions were computed for water confined in an all-silica LTA (red), for bulk water (black) and for a single gas phase water molecule (green). 


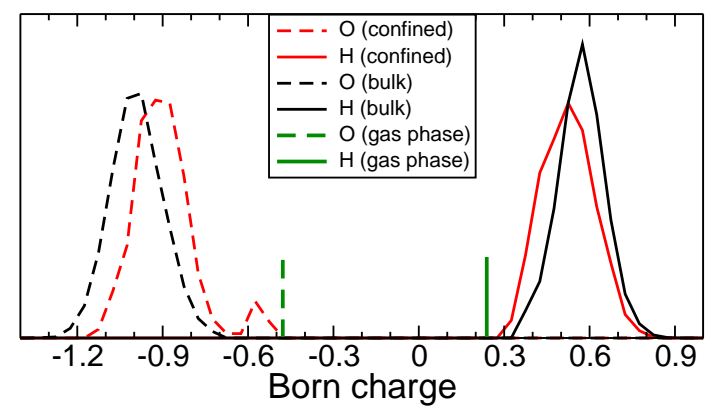

Figure 16: Distribution of partial charges of the water oxygen and hydrogen atoms according to the Born population analysis methods. The distributions were computed for water confined in an all-silica LTA (red), for bulk water (black) and for a single gas phase water molecule (green). 